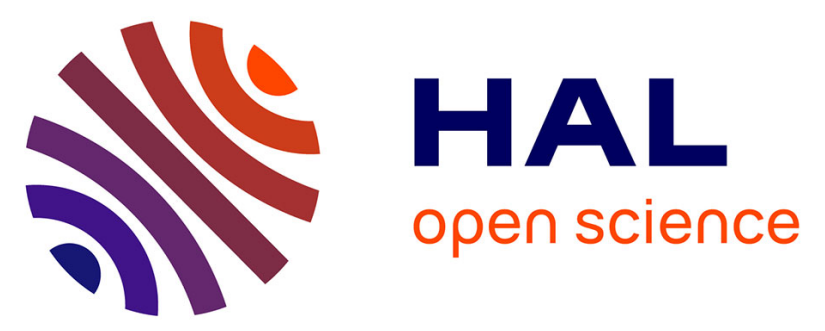

\title{
Joint scheduling of jobs and preventive maintenance operations in the flowshop sequencing problem: A resolution with sequential and integrated strategies.
}

Fatima Benbouzid-Sitayeb, Christophe Varnier, Noureddine Zerhouni, Sid Ali Guebli, Yassine Bessadi

\section{To cite this version:}

Fatima Benbouzid-Sitayeb, Christophe Varnier, Noureddine Zerhouni, Sid Ali Guebli, Yassine Bessadi. Joint scheduling of jobs and preventive maintenance operations in the flowshop sequencing problem: A resolution with sequential and integrated strategies.. International Journal of Manufacturing Research, 2011, 6 (1), pp.30-48. 10.1504/IJMR.2011.037912 . hal-00720671

\section{HAL Id: hal-00720671 \\ https://hal.science/hal-00720671}

Submitted on 25 Jul 2012

HAL is a multi-disciplinary open access archive for the deposit and dissemination of scientific research documents, whether they are published or not. The documents may come from teaching and research institutions in France or abroad, or from public or private research centers.
L'archive ouverte pluridisciplinaire $\mathbf{H A L}$, est destinée au dépôt et à la diffusion de documents scientifiques de niveau recherche, publiés ou non, émanant des établissements d'enseignement et de recherche français ou étrangers, des laboratoires publics ou privés. 


\title{
Joint scheduling of jobs and preventive maintenance operations in the flowshop sequencing problem: A resolution with sequential and integrated strategies
}

\author{
${ }^{1}$ Fatima BENBOUZID-SITAYEB*, Sid Ali GUEBLI, Yassine BESSADI \\ ${ }^{2}$ Christophe VARNIER, Nourredine ZERHOUNI.
}

\begin{abstract}
Usually, scheduling of maintenance operations and production sequencing are dealt with separately in the literature and, therefore, also in the industry. Given that maintenance affects available production time and elapsed production time affects the probability of machine failure, this interdependency seems to be overlooked in the literature. This paper presents a comparative study on joint production and preventive maintenance scheduling strategies regarding flowshop problems. The sequential strategy which consists of two steps: first scheduling the production jobs then inserting maintenance operations, taking the production schedule as a strong constraint. The integrated one which consists of simultaneously scheduling both maintenance and production activities based on a common representation of these two activities. For each strategy, a constructive heuristic and two meta-heuristics are proposed: NEH heuristic, Genetic algorithm and Taboo search. The goal is to optimize an objective function which takes into account both production and maintenance criteria. The proposed heuristics have been applied to non-standard test problems which represent joint production and maintenance benchmark flowshop scheduling problems taken from Benbouzid et al. (2003). A comparison of the solutions yielded by the heuristics developed in this paper with the heuristic solutions given by Taillard (1993) is undertaken with respect to the minimization of performance loss after maintenance insertion. The comparison shows that the proposed integrated GAs are clearly superior to all the analyzed algorithms.
\end{abstract}

KEYWORDS: Production, Preventive maintenance, Joint scheduling, GA, Taboo search, NEH heuristic, Flowshop.

\section{Introduction}

Scheduling is one of the most significant activities of the control system since it aims at planning orders on machines in order to ensure customer satisfaction (through the respect of the due dates) and system profitability (through an efficient use of the resources). One of the most frequent production scheduling problems is the flowshop problem (FSP). This problem implies a natural ordering of the machines in the shop, in such a way that each job has one operation on each machine and all jobs have the same ordering sequence on each machine. At any time, each machine can process at most one job and each job can be processed on at most one machine. Preemption is not allowed. Here we consider the permutation FSP (PFSP), i.e. the same job order is chosen on every machine. The objective is then to find a permutation of jobs that minimizes the completion time $\mathrm{C}_{\max }$, also called makespan, of the last job. Conventionally, this problem is denoted by $\mathrm{n} / \mathrm{m} / \mathrm{P} / \mathrm{C}_{\max }$. It is a well-known NP-hard problem (Rinnooy, 76).

Another task closely related to production scheduling in industrial settings is maintenance. However, both activities conflict since they act on the same resources (equipments). As it is known, maintenance operations consume production time whereas delaying maintenance operations because of production demands may increase the probability of machine failure. Despite the relationship between them in industrial settings, scheduling of maintenance operations and production sequencing are dealt with separately in the production systems and operations research literature. Our contention is that manufacturing system productivity would benefit from considering jointly scheduling and maintenance to find the best compromise between the maintenance cost and the risk of machines unavailability.

In this paper, we discuss the joint scheduling of independent jobs and maintenance in the flowshop sequencing problem, which we denoted JPMSP (Joint Production and Maintenance Scheduling Problem), with no pre-emption, no machine breakdowns and no setup times. The machine ready time and job release times are all zeros. The objective is then to optimise an objective function which takes into account both maintenance and production criterion. A constructive heuristic and two meta-heuristics are proposed: NEH heuristic (Nawaz et al., 1983), Genetic algorithm (GA) (Golberg, 1989) and Taboo Search (TS) (Glover, 1989), according to the most interesting joint scheduling strategies: sequential and integrated ones. This approach is inspired by the work of Ponnambalam et al. (2001) where an attempt has been made to evaluate the different constructive heuristics and a genetic algorithm to solve FSP. Among all the constructive heuristics compared, NEH heuristic performs well and gives minimum values for most of the problems. However, GA performs better then heuristics for FSP. We include TS in this study to cover the space of resolution methods.

* Corresponding author 
The remainder of this paper is organized as follows. We introduce the background of JPMSP with both theoretical and practical aspects of joint production and maintenance scheduling in section 2. Section 3 introduces the topic and the basic concepts on maintenance and the objective functions to optimize. Section 4 is devoted to joint maintenance and production scheduling strategies in permutation flowshop. Constructive heuristic and two meta-heuristics are proposed. Computational experiences with the proposed algorithms are reported in the section 5. A set of non-standard test problems which represent joint production and maintenance benchmark flowshop scheduling problems, are used for this purpose. Finally, the last section contains conclusion regarding the computational experience followed by suggested future research.

\section{The background of JPMSP}

As in industry, production scheduling and maintenance planning are typically treated independently in the production systems and operations research literature. On one hand, an extensive amount of this research addresses production/machine scheduling without worrying about maintenance. On the other hand the literature on the use of mathematical modelling for analyzing, planning and optimizing maintenance actions is also extensive. However, the interaction between maintenance and production, particularly their joint scheduling, is relatively little studied and rather recent in the literature.

There are a few studies that consider combining production scheduling and maintenance planning. These studies' aim is to determine the best moment to plan maintenance operations according to a compromise between the maintenance cost and the risk of machines unavailability (Brandolese et al., 1996; Sanmarti et al., 1997). Some authors propose to solve the single-machine scheduling problem while others propose to solve it on $\mathrm{m}$ machines. Graves and Lee (1999) formulate the single-machine problem with total weighted completion time as the objective function, but they schedule only one maintenance operation during the planning horizon. Qi et $a l$. (1999) studied scheduling jobs on a single machine problem with the possibility of multiple maintenance actions. The time elapsed between two maintenance actions can not exceed a given value, but they do not explicitly model the risk of not performing maintenance. Raza et al. (2007) considered joint scheduling of jobs and preventive maintenance operations on a single machine with an objective to minimize the total earliness and tardiness of jobs about a common due date (see also Bennour et al., 2001; Kaabi et al., 2002; Lee and Lin, 2001; Sortrakul et al., 2005). Ashayeri et al. (1996) use a discrete-time multi-machine integrated model, but they consider lot sizing rather than scheduling individual jobs. Lee and Chen (2000) extend the work of Graves and Lee (1999) to parallel machines, but still with only one maintenance action, see also (Harrath and ChebelMorello, 2002; Kaabi et al., 2003).

There are only a few studies that explicitly try to integrate flowshop scheduling and maintenance decisions by optimizing them simultaneously. Cassady and Kutanoglu (2003) is one of the few cases where an integrated model for job and preventive maintenance activities scheduling is shown such that total tardiness is minimized. However, the authors propose a total enumeration approach that is tested against very small problems with a maximum of eight jobs which is not practical. A more complex hybrid flowshop problem is tackled in Allaoui and Artiba (2004), but in this work, preventive maintenance operations are given as problem data and considered as constraints, i.e., not as a result of the machine operation like we consider in this work. Aggoune (2004) minimize the makespan of a flowshop with availability constraints. Two maintenance policies are considered. In the first, maintenance starting times are fixed, where as in the second maintenance must be performed within a given time window. Ruiz et al. (2006) propose two preventive maintenance policies, and then describe a new criterion to schedule preventive maintenance operations to the production sequence. Six adaptations of heuristics and metaheuristics methods that include the proposed criterion have been scheduled.

All the works listed above study the problem in a very restricted way and are limited, most of the time, to only one machine or only one scheduling strategy. Moreover, they are interested only in production criteria even for the cases where the unavailability periods are due to preventive maintenance interventions. While this body of work is very important, it is difficult to apply in practice and what we propose in this paper is a simpler way of considering preventive maintenance operations scheduling. Our approach consists in combining scheduling resolution methods and joint scheduling strategies aiming to describe the benefits of integrating the two activities into a decision-making process.

\section{Scheduling and preventive maintenance}

Maintenance is understood as any activity carried out on a system to maintain it or to restore it to a specific state (Boroloni, 2004). Maintenance operations can be classified into two large groups according to the intervention time: Corrective Maintenance (CM) and Preventive Maintenance (PM). CM is carried out when the failure has already taken place. PM consists in carrying out the operations in machines and equipment before the failure or the breakdown takes place, and at fixed time intervals previously established. The objective of PM is to prevent failures before they happen and therefore to minimize the probability of failure. 
The importance of PM is well established in a manufacturing environment. A sound PM program results in reducing CM cost and cost of defective production. It also increases the availability of the production facility. The choice of PM, for this study, is a consequence of its planned aspect which makes it the most adapted for the maintenance scheduling. In this case, the search for a production schedule will be correlated to the search for PM planning which is predefined and easy to implement.

One accounts in the literature two joint production and PM scheduling strategies based on a different policy (Lee and Chen, 2000) aiming to solve conflicts between production and maintenance: sequential strategy and integrated strategy. The sequential strategy consists of scheduling production jobs, then inserting PM operations, taking the production schedule as an additional constraint of resources unavailability in the resolution of the problem. The integrated one consists of simultaneously scheduling both maintenance and production activities based on a common representation of these two activities. Such policy limits the risks of conflicts, and thus makes it possible to optimize a joint objective function (Brandolese et al., 1996; Sloan et al., 2000).

The PM can be stated as follows. Each machine is maintained periodically at known intervals of time. The PM operations are periodic interventions occurring every $\mathrm{T}$ periods and each occurrence depends on the ones preceding it on the same machine. The processing time of PM operation $\mathrm{j}$ on machine $\mathrm{i}$ is $\mathrm{p}_{\mathrm{ij}}{ }_{\mathrm{ij}}$. The processing times are fixed, nonnegative and evaluated with more or less certainty. Moreover, the periodicity $\mathrm{T}$ of these operations can vary in a tolerance interval noted [Tmin, Tmax]. This interval gives some flexibility to plan PM operations while respecting the production constraint, disturbing the least possible the production schedule, and respecting the maintenance equipment periodicity.

Ideally a PM operation is planed inside the interval [Tmin, Tmax] (Figure 1-Area 2). If the period is lower than Tmin (Figure 1-Area 1), PM interventions will be too frequent, compared to the machine real need, and thus induce a too high maintenance cost. Moreover, if some PM operations are programmed after Tmax (Figure 1-Area 3), breakdowns are likely to appear and consequently CM operations too. As a result, the machine would be unavailable, and also induces a maintenance cost increase. A machine i can be subject to several different PM operations which will be repeated periodically. Let:

- $\mathbf{M}_{\mathrm{ij}}$ : the PM operation $\mathrm{j}$ on machine $\mathrm{i}$.

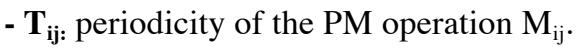

- $\operatorname{Tmin}_{\mathrm{ij}}$ : earliest time separating two consecutives occurrences of $\mathrm{M}_{\mathrm{ij}}$;

- Tmax $_{\mathrm{ij}}$ : latest time separating two consecutives occurrences of $\mathrm{M}_{\mathrm{ij}}$;

- $\mathbf{p}_{\mathrm{ij}}$ : processing time of PM operation $\mathrm{M}_{\mathrm{ij}}$. It is supposed to be known and constant.

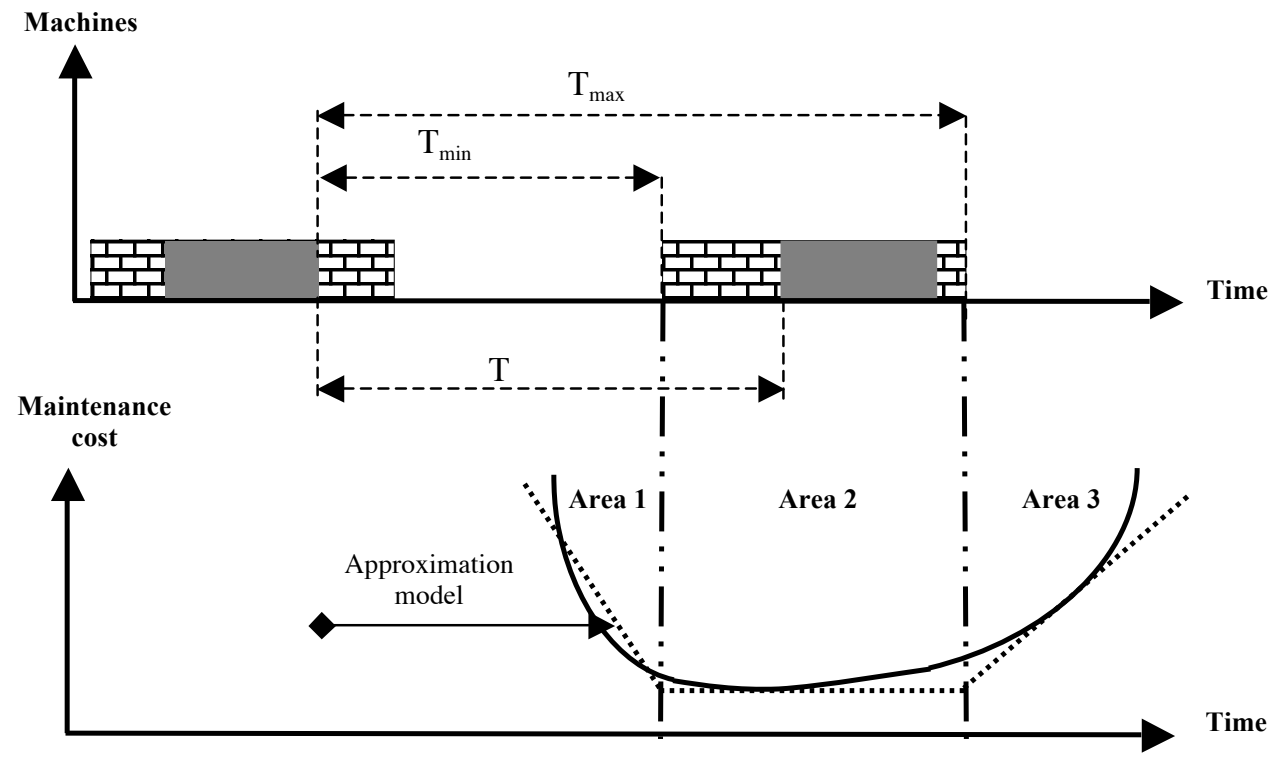

Figure 1: Tolerance interval of a PM operation.

Ideally a PM operation is programmed inside the interval $\left[\mathrm{T}_{\min }, \mathrm{T}_{\max }\right]$. However, it can be programmed before $\mathrm{T}_{\min }$ and thus be considered in advance (this advance is noted $\mathrm{E}^{\prime}$ ), or after $\mathrm{T}_{\max }$ and thus be considered as late (this delay is noted L'). Earliness and tardiness of the $\mathrm{k}^{\text {th }}$ occurrence of the PM operation $\mathrm{M}_{\mathrm{ij}}$ is computed as follows:

- $\mathbf{t}^{\prime}{ }_{\mathrm{ijk}}$ : execution time of the $\mathrm{k}^{\text {th }}$ occurrence of $\mathrm{M}_{\mathrm{ij}}$.

- $\mathbf{E}_{\mathrm{ijk}}$ : Earliness execution time of the $\mathrm{k}^{\text {th }}$ occurrence of $\mathrm{M}_{\mathrm{ij}}$.

$E^{\prime}{ }_{i j k}=\max \left(0, t^{\prime}{ }_{i j k}+p^{\prime}{ }_{i j}+\operatorname{Tmin}_{i j}-t^{\prime}{ }_{i j k-1}\right)$ 
- $\mathbf{L}_{\mathrm{ijk}}:$ Tardiness execution time of the $\mathrm{k}^{\mathrm{th}}$ occurrence of $\mathbf{M}_{\mathrm{ij}}$. $L^{\prime}{ }_{i j k}=\max \left(0, t^{\prime}{ }_{i j k-1}-t^{\prime}{ }_{i j k}-p^{\prime}{ }_{i j}-\operatorname{Tmax}_{i j}\right)$

The tolerance interval of the $\mathrm{k}^{\text {th }}$ occurrence of $\mathrm{PM}$ operation $\mathrm{M}_{\mathrm{ij}}\left[\operatorname{Tmin}_{\mathrm{ijk}}, \operatorname{Tmax}_{\mathrm{ijk}}\right]$ is given by:

- $\boldsymbol{T}_{\boldsymbol{m i n}}{ }_{i j k}=t^{\prime}{ }_{j} k_{-1}+p^{\prime}{ }_{i j k}+T_{\text {minij }}$;

- $\boldsymbol{T m a x}_{i j k}=t^{\prime}{ }_{i j} k_{-1}+p^{\prime}{ }_{i j k}+T_{\text {maxij }}$.

The goal of joint scheduling is to propose a method that provides a common planning for production jobs and PM operations. Thus, the objective is to optimize a common objective function which takes into account both production and maintenance criterion. The constraints imposed by the customers to their suppliers are often expressed in term of time, which lead us naturally to the minimization of the makespan. One will note $f_{1}$ the production objective function:

$$
f_{1}=C_{\max }=\operatorname{Max}\left(c_{i j}\right)^{1}
$$

We introduced in figure 1 a cost curve that depends on the period where PM operations are executed (time). This curve can be established on the base of a mathematical model. The purpose of this work is to optimize a time and not a cost. From the supplier point of view, the respect of the maintenance periods influences the constraints of the production system. One will note $\mathrm{f}_{2}$ the maintenance objective function.

$$
f_{2}=\sum_{j=1}^{m} \sum_{k=1}^{M a x j} E_{j k}^{\prime}+L^{\prime}{ }_{j k}^{2}
$$

To optimize the two criteria, we take into account the following objective function:

$$
f=w_{1} f_{1}+w_{2} f_{2}
$$

$\left(\mathrm{w}_{1}, \mathrm{w}_{2}\right)$ are weights which will measure the respective contributions of production and maintenance in the global objective function. The goal is not to study multicriteria optimization but only to measure the impact of production or maintenance on the global objective function. For that the parameters $\mathrm{w}_{1}$ and $\mathrm{w}_{2}$ can depend on the number of tasks, processing time or problem size. They are independent and non complementary. They are fixed by the user according to the degree of importance given to production or maintenance.

\section{Proposed solution algorithms}

We propose three heuristic algorithms to solve JPMSP. The first algorithm is NEH heuristic. The other two algorithms are metaheuristics based on TS and GA algorithms. We discuss the most salient aspects of the adaptation of the three proposed algorithms, in the case of sequential and integrated strategies, briefly as follows.

\subsection{Sequential strategy}

The first step of sequential strategy consists first in generating a production schedule with GA, TS and NEH. Then PM operations are inserted in the generated schedule. The parameters setting adopted for GAs and NEH heuristic are those used in the research study by Ponnambalam et al. (2001). For TS, we use the application of this technique proposed by Nowicki and Smutnicki (1996). The complete details are not reported for the sake of concise presentation. Moreover, as we deal with PFSP, the insertion of the PM operations will be done first on single machine, then on the rest of the machines (m-1), as described below.

\subsubsection{Resolution of the single-machine problem}

The insertion of PM operations on one machine will be done according to two heuristics: Basic and In-depth search. These heuristics are presented below.

(a) The Basic Heuristic (BH) (Kaabi et al., 2002) consists in inserting a PM operation exactly at $\mathrm{T}$ (period for a PM operation insertion), $\mathrm{T}_{\min }$ or $\mathrm{T}_{\max }$ (resp. earliest and latest period for a PM operation insertion). If a production job is planned at this time, the PM operation will be deferred and will start only at the completion time of the production job.

(b) The In-depth search Heuristic (IH) (Benbouzid et al., 2003) takes into account the tolerance interval ( $\S$ 3) (Figure 2). A PM operation can be inserted on all the sites of its tolerance interval, i.e. at the completion time of each production job which is inside this interval; or exactly at $\mathrm{T}_{\min }$ if no job is executed at this time.

\footnotetext{
${ }^{1} \mathrm{c}_{\mathrm{ij}}$ : completation time of task $\mathrm{j}$ on the machinei.

${ }^{2}$ Maxj represents the effective occurrence number of the PM operation $\mathrm{M}_{\mathrm{j}}$.
} 
Moreover, it is possible to use one of the two strategies described above: (S1) PM operations advances and delays are not allowed. PM operations are inserted only in their tolerance interval or (S2) PM operations advances and delays are allowed. A PM operation can be inserted just before $\mathrm{T}_{\min }$ or just after $\mathrm{T}_{\max }$.

For each site, one evaluates the obtained schedule (global objective function $\mathrm{f}$ ). The best site is the one minimizing the function $\mathrm{f}$. if a PM operation is inserted before $\mathrm{T}_{\min }$ (maintenance useless) or after $\mathrm{T}_{\max }$ (risk of breakdown) provided that the evaluation of the resulting scheduling is better. The search for a solution, in the case of one machine is done in-depth initially without feed-back.

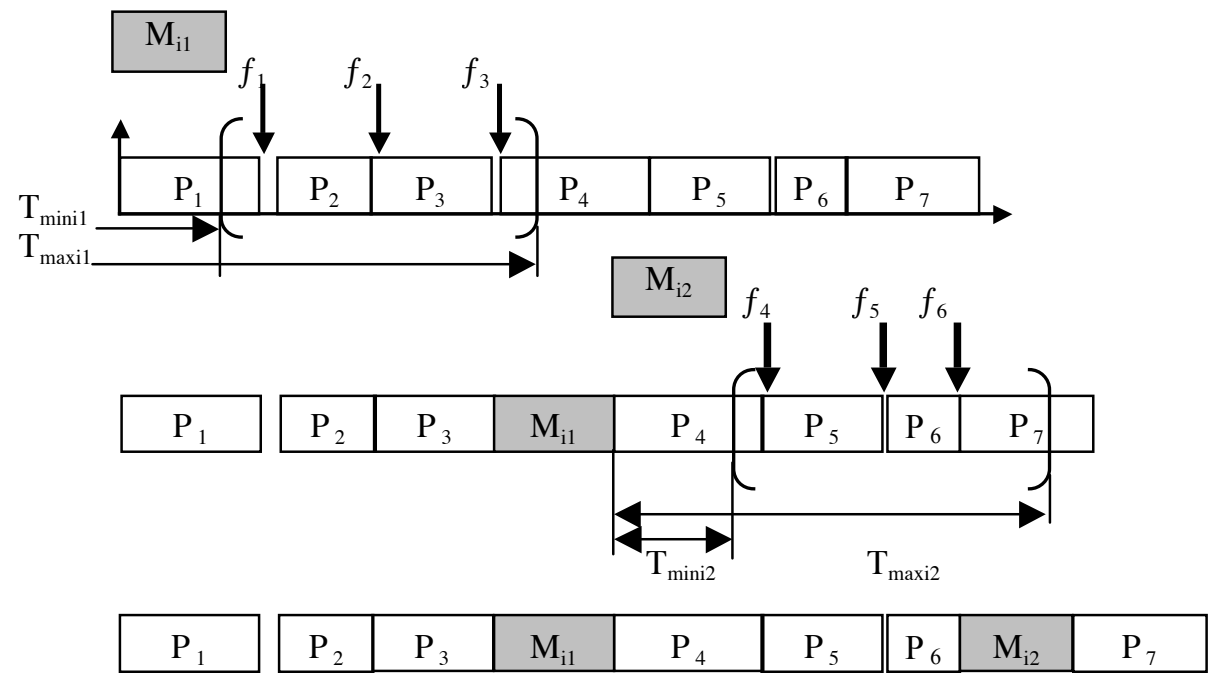

Figure 2: Possible sites of a PM operation insertion.

\subsubsection{Resolution of the multi-machines problem}

The insertion of PM operations on all machines will be done according to three heuristics introduced by Benbouzid et al. (2003): Naive, Ascending and Descending heuristics. These three heuristics are presented below.

(a) Naïve Heuristic (NH) inserts all PM operations, on all machines, from the first until the last one. The insertion of PM operations on one machine is done according to $\mathrm{BH}$ heuristic developed in the preceding section.

(b) Ascending Heuristic (AH) inserts PM operations, on all machines, from the first until the last one. The insertion of PM operations on one machine is done according to $\mathrm{IH}$ heuristic developed in the preceding section.

(c) Descending Heuristic (DH) inserts PM operations, on all machines, from the last until the first one. The insertion of PM operations on one machine is done according to IH heuristic.

\subsection{Integrated strategy}

In the sequential strategy, the objective function to optimize is mainly the production one $\left(\mathrm{C}_{\max }\right)$. In the integrated one the aim is to optimize a global objective function which takes into accounts both production and maintenance criterion. Therefore, the resolution with the integrated strategy is based on a joint representation of production and maintenance data. In what follows, we will first, present the proposed representation of a joint scheduling solution, then for each heuristic (GA, TS and NEH), the proposed adaptations.

\subsubsection{Joint production and PM schedule representation}

A joint production and maintenance solution is coded as a structure with two fields: the first one is a vector $\mathrm{S}$ that represents the execution sequence of production jobs. The second one is a matrix $M$ that represents the sites of PM operations insertion on the machines. The element $M[i, j]$ represents the insertion of the $\mathrm{j}^{\text {th }} \mathrm{PM}$ operation of the $i^{\text {th }}$ machine in the sequence $\mathrm{S}$.

Example:

Production sequence $S$

\begin{tabular}{|l|l|l|l|l|l|l|l|l|l|}
\hline 1 & 9 & 3 & 8 & 5 & 6 & 7 & 4 & 2 & 0 \\
\hline
\end{tabular}

Matrix $M$

$$
\left[\begin{array}{llll}
0 & 1 & 4 & 6 \\
1 & 2 & 5 & \\
0 & 4 & 7 & 8
\end{array}\right]
$$


$\mathrm{S}=(1 ; 9 ; 3 ; 8 ; 5 ; 6 ; 7 ; 4 ; 2 ; 0)$

$\mathrm{S}[2]=9 ; \mathrm{S}[6]=6 ;$ etc...

$\mathrm{M}[3,2]=4$ means that the second PM operation on the third machine is inserted in position 4 (after the production job 8) in sequence $\mathrm{S}$. The execution order of PM operations on the three machines according to the preceding example is the following:

Machine $1: \mathrm{M}_{01}, \mathrm{P}_{1}, \mathrm{M}_{11}, \mathrm{P}_{9}, \mathrm{P}_{3}, \mathrm{P}_{8}, \mathrm{M}_{21}, \mathrm{P}_{5}, \mathrm{P}_{6}, \mathrm{M}_{31}, \mathrm{P}_{7}, \mathrm{P}_{4}, \mathrm{P}_{2}, \mathrm{P}_{0}$

Machine 2: $\mathrm{P}_{1}, \mathrm{M}_{02}, \mathrm{P}_{9}, \mathrm{M}_{12}, \mathrm{P}_{3}, \mathrm{P}_{8}, \mathrm{P}_{5}, \mathrm{M}_{22}, \mathrm{P}_{6}, \mathrm{P}_{7}, \mathrm{P}_{4}, \mathrm{P}_{2}, \mathrm{P}_{0}$

Machine 3: $\mathrm{M}_{03}, \mathrm{P}_{1}, \mathrm{P}_{9}, \mathrm{P}_{3}, \mathrm{P}_{8}, \mathrm{M}_{13}, \mathrm{P}_{5}, \mathrm{P}_{6}, \mathrm{P}_{7}, \mathrm{M}_{23}, \mathrm{P}_{4 \mathrm{~s}}, \mathrm{M}_{33}, \mathrm{P}_{2}, \mathrm{P}_{0}$

\subsubsection{An integrated genetic algorithm}

In this section, we discuss the detailed features of the proposed Integrated Genetic Algorithm (IGA). The new GA operators used have the particularity of working on a joint production and PM individual. These operators are described as follows:

(a) Crossover. Crossover can be applied on the production sequence $\mathrm{S}$ or the maintenance matrix $\mathrm{M}$. We propose the following crossover:

- Production crossover. They are the classical crossover on production defined for GA (Goldberg, 1989; Portmann, 1996).

- Maintenance crossover. Benbouzid et al. propose in 2006 a new maintenance crossover called kpoints horizontal crossover.

(b) Mutation. Also mutation can be done on production sequence S or maintenance matrix M. We propose the following mutation:

- Production mutation: In Taillard (1990) it was shown that the neighbourhood based on insertionmoves can be evaluated more efficiently than the one based on interchange-moves and additionally gives at least the same solution quality. Thus, we applied the insertion-moves only to the production sequence $S$ while keeping the maintenance matrix $\mathrm{M}$ unchanged. The goal of this strategy is to create new individuals by changing the execution order of the production jobs, while keeping PM operations in their initial sites.

- Maintenance mutation: Benbouzid et al. (2006) propose two new mutations on maintenance called random mutation and k-points vertical mutation.

At each iteration, in order to diversify the generated population, we apply randomly one of the news operators presented above.

\subsubsection{Integrated TS}

In this section, we discuss the detailed features of the proposed Integrated Taboo Search (ITS) algorithm as follows:

(a) Initial solution. Initial solution is any sequence of jobs that satisfies the preventive maintenance requirement. TS can start the search with a random solution, or a provided one. For us, a solution can be complete or partial. A complete solution represents a joint production and PM scheduling solution. In this case, a complete initial solution is obtained after insertion of PM operations using one of the heuristics presented in the preceding section, on a production schedule generated randomly.

\section{(b) Neighbourhood}

The move from a solution to another in the neighbourhood can be done by mutation in the production sequence, the PM matrix, or on both at the same time. We defined two types of moves allowing generating news solutions in the neighbourhood of the current one. The first one concerns production jobs, and the second one PM operations. The neighbour solution is chosen according to one of the following strategies: Best move, First improve or Randomly (Goldberg, 1989).

- Production. A neighbourhood solution is obtained by swapping two randomly selected jobs, in the production sequence $\mathrm{S}$, while the maintenance matrix $\mathrm{M}$ stays unchanged.

- Maintenance. A PM operation has several possible insertion sites in its tolerance interval. It is, thus, interesting to define the neighbourhood of a solution as all the possible insertion sites for one or more PM operations, on one or more machines.

In order to diversify the search, when generating the neighbourhood $\mathrm{N}\left(\mathrm{S}_{\mathrm{c}}\right)$ of the current solution $\mathrm{S}_{\mathrm{c}}$, we apply randomly one of the mutation operators presented above.

(c) Candidate list size: It is a list containing a subset of neighbourhood moves examined. A candidate list 
size of 20 is selected, for each iteration, after using the conclusions from a series of tests performed in Nowicki and Smutnicki (1996).

(d) Taboo restriction: In our implementation, attributes of a schedule are jobs swapped in a schedule which are recorded in taboo list. The taboo list can store a maximum of 7 moves and it is updated using First In First Out (FIFO) strategy.

- Aspiration criterion: It is satisfied when the best neighbour solution of the current iteration is found better than the best solution visited so far.

- Stopping criterion: The algorithm is stopped after 5000 iterations of no improvement.

\subsubsection{Integrated $\mathrm{NEH}$}

An integrated resolution of JPMSP with NEH (INEH) requires the definition of a preceding order among all the tasks to be ordered (production and maintenance ones). This order is clearly defined among the production jobs (the job with the highest processing time on all machines should be placed first), in the case of makespan minimization. For PM operations, the order is preset on each machine (each PM operation depends on the ones preceding it on the same machine). The problem of both production jobs and PM operations order remains. We propose the following strategy: First, the production jobs are sorted in decreasing order according to their total processing time on the machines (Sorted list L). PM operations are sorted too, in increasing order, according to their $\mathrm{T}_{\min }$ (Sorted list L'). Then the first two jobs (those with largest processing times) and the first occurrence of each PM operations with earliest $\mathrm{T}_{\min }$ are taken, and all the possible schedules including these production jobs and PM operations are evaluated, while respecting the constraint of PM operations insertion. The sequence with the best objective function value is taken for further consideration. For every remaining job in the list L and PM operation in the list L', we find the best schedule by inserting the production job at all possible sites in the sequence of jobs that are already scheduled and the PM operations at all possible sites inside their tolerance interval.

\section{Computational results and analysis}

In this section, we present the results of a series of computational experiments conducted to test the effectiveness of the proposed heuristics (NEH, TS and GA), in the resolution of JPMSP with sequential and integrated strategies in the flowshop sequencing problem. For each strategy, we report on two sets of experiments. In the first set we have undertaken a sensitivity analysis of performance of the new parameters proposed. The complete details are reported in the next section. The second set analysis's the effectiveness of the proposed heuristics.

To our knowledge, there is no instance for JPMSP. Therefore, the test problems for evaluating the proposed heuristics are non-standard ones, generated by following the procedure given by Benbouzid et al. (2003) for generating joint production and preventive maintenance benchmark flowshop scheduling problems. The production benchmarks are those of Taillard (1993). Thaillard has produced a set of problems for PFSP to minimize the makespan. For PM, Benbouzid et al. (2003) developed a processor to generate randomly PM benchmarks. The used parameters are the number of machines and PM parameters ( $\mathrm{p}, \mathrm{T}_{\mathrm{T}} \mathrm{T}_{\min }$ and $\mathrm{T}_{\max }$ ). They generate only one PM type per machine for each problem. Moreover, the processing time of a PM operation is identical for all its occurrences. These ones are combined to production benchmarks to obtain joint production and maintenance benchmarks. The objective functions taken into consideration are the minimization of makespan for the production, the minimization of the sum of the delays and advances for maintenance $(\S 3)$, and finally the minimization of makespan after maintenance insertion for joint production and maintenance scheduling. The contribution of production and maintenance objective functions in the common objective function $\mathrm{f}$ are equal to $1\left(\mathrm{w}_{1}=1\right.$ and $\left.\mathrm{w}_{2}=1\right)$. The proposed common weighted global objective function will allow tackling the problem in a simplified way.

One difficulty faced by researchers in scheduling is to compare their developed heuristics with those of other researchers. If the standard set of test problems is accessible, different algorithms' performances can be compared on exactly the same set of test problems. In the case of JPMSP, few computational results can be found and no optimal or near optimal results exists. Since no competitive results for JPMSP are found in the literature, we compare our results with the upper bound values of makespan yielded by Taillard (1993) according to the performance loss after maintenance insertion. Thus, this loss is as an evaluation parameter for the proposed adaptation of NEH heuristic, TS and GA. When comparing the solutions, the best one is the one which gave the least increase. 


\subsection{Sequential strategy}

In this section, we present an evaluation of the results obtained with NEH, TS and GA in the resolution of JPMSP by the sequential strategy. We report on two sets of experiments: the first one concerns the production scheduling and the second one is an analysis of performance of the maintenance insertion heuristics.

For the first set of experiments, it is to be noted that we have undertaken a sensitivity analysis of performance for GA and TS by varying different parameters such as those involved in the choice of the number of generation and the neighbourhood size. These parameters were experimentally found to be good and robust for the problems tested. The complete details are not reported for the sake of concise presentation. For TS and GA the following parameter settings were used:

- TS was executed with the following parameters: Number of generations: 5000; neighbourhood size: 50; Taboo list size: 7 . For each benchmark, the best result is retained.

- The results of GA were obtained after several executions of the method. The best result is saved, as well as the associated parameters. The following parameters are the same for all the executions of GA: Number of generations: 5000; crossover rate: 0.7 ; mutation rate: 0.01 ; $\mathrm{N}$ best, the replacement is done between the selected population and the crossed one. Population size: between 50 and 100 . However, since the GA are known to prematurely converge, the only way to get the best solution was to use a strategy called "sharing" (Bonabeau, 1999). This strategy allows diversifying the population, avoiding GA to get trapped in a local optimum. We use sharing with the following parameters: $\alpha_{\text {Sharing }}=0.99$ and $\beta_{\text {Sharing }}=4$.

For the second set of experiments, table 1 presents the results of the evaluation of the maintenance insertion heuristics on production sequence. We test on each production sequence, the maintenance insertion heuristics developed in section 4.1. By considering the objective of minimizing the performance loss after maintenance insertion, we evaluate the maintenance insertion heuristics in comparison with the upper bound values of makespan presented by Taillard (1993) for 90 benchmark permutation flowshop scheduling problems taken from Taillard (1993). We retained the best result for the chart. The maintenance insertion heuristics considered are $\mathrm{NH}, \mathrm{AH}$ and $\mathrm{DH}$, as presented in section 4.1. The following abbreviations are used:

- $\mathrm{NH}$ heuristic at $\mathrm{T}, \mathrm{T}_{\min }$ and $\mathrm{T}_{\max }$ : respectively $\mathrm{NH}, \mathrm{NH}_{\min }$ and $\mathrm{NH}_{\max }$;

- AH heuristic with and without PM operations advances or delays: respectively $\mathrm{AH}+$ and $\mathrm{AH}-$;

- DH heuristic with and without PM operations advances or delays: respectively DH+ and DH-.

Table 1. Results of the evaluation of maintenance insertion heuristics ${ }^{3}$

\begin{tabular}{cccccccc}
\hline \hline Heuristics & \multicolumn{3}{c}{$\mathrm{NH}$} & \multicolumn{2}{c}{$\mathrm{DH}$} & \multicolumn{2}{c}{$\mathrm{AH}$} \\
\hline \multirow{2}{*}{ Best results } & $\mathrm{NH}$ & $\mathrm{NH}_{\min }$ & $\mathrm{NH}_{\max }$ & $\mathrm{DH}+$ & $\mathrm{DH}-$ & $\mathrm{AH}+$ & $\mathrm{AH}-$ \\
\cline { 2 - 8 } & $0 \%$ & $0 \%$ & $4 \%$ & $21 \%$ & $12 \%$ & $40 \%$ & $23 \%$ \\
\hline Total & & $4 \%$ & & & $33 \%$ & \multicolumn{2}{c}{$63 \%$} \\
\hline \hline
\end{tabular}

According to the results of table 1, one can notice that both ascending (AH) and descending (DH) heuristics which tolerate advances and delays of PM operations perform better (respectively $40 \%$ and $21 \%$ ) than those which do not tolerate such advances and delays (respectively $23 \%$ and $12 \%$ ). The main reason for such a superior performance is due to the fact that a PM operation has more possible insertion sites in the first heuristics $(\mathrm{AH}+$ and $\mathrm{DH}+)$ than in the second ones $(\mathrm{AH}-$ and $\mathrm{DH}-)$. Indeed, when PM operations advances and delays are allowed, these ones can be inserted before their $T_{\min }$ or after their $T_{\max }$, which implies more possible insertion sites for PM operations.

The worst result is obtained by NH heuristic with only $4 \%$ of the best results. That is mainly due to the $\mathrm{NH}$ constraint that consists in inserting PM operations at only their $\mathrm{T}, \mathrm{T}_{\min }$ or $\mathrm{T}_{\max }$ periods which implies to defer PM operation after the completion time of production job, if these ones are planed at this time. As a result, the quality of the joint schedule is low. Releasing this constraint (tolerating advances and delays of PM operations), increases the joint schedule quality.

Based on the results of table 1, we used $\mathrm{AH}+$ heuristic to insert PM operations in the production sequence. The results after insertion of PM operations in the production sequences generated at the preceding phase are presented in table 2 .

\footnotetext{
${ }^{3}$ The results of table 1 represent the number of times an insertion heuristic gave the best results, among all the tested benchmarks.
} 
Table 2: Joint production and PM scheduling with sequential strategy

\begin{tabular}{lllllll}
\hline \hline & \multicolumn{2}{l}{ Sequential (Production) } & & \multicolumn{2}{l}{ Sequential (After maintenance insertion) } \\
\hline Benchmark & NEHprod $^{4}$ & TSprod & GAprod & NEHseq $^{5}$ & TSseq & GAseq \\
\hline $7 \times 7$ & 6590 & 6590 & 6590 & 7090 & 7409 & 6925 \\
\hline $8 \times 8$ & 8566 & 8364 & 8457 & 8988 & 9235 & 8580 \\
\hline $20 \times 5$ & 1303 & 1249 & 1318 & 1252 & 1262 & 1202 \\
\hline $20 \times 10$ & 1603 & 1602 & 1583 & 1700 & 1650 & 1640 \\
\hline $20 \times 15$ & 2002 & 2145 & 1972 & 2200 & 2209 & 2166 \\
\hline $30 \times 15$ & 2644 & 2702 & 2643 & 2867 & 2849 & 2796 \\
\hline $50 \times 10$ & 3153 & 3271 & 3218 & 3696 & 3356 & 3189 \\
\hline $75 \times 20$ & 5465 & 5497 & 5227 & 5852 & 5768 & 7122 \\
\hline $100 \times 10$ & 530 & 577 & 533 & 552 & 647 & 556 \\
\hline \hline
\end{tabular}

The results obtained with GA represent $80 \%$ of the best results, $20 \%$ are given by TS. Heuristic NEH performed better for large-sized problems. We can conclude that GA are better than TS and heuristic NEH for the sequential strategy.

One will note from Table 2 that some good results, when optimising production scheduling (first step of the sequential strategy), became worst after maintenance insertion (second step of the sequential strategy). Indeed, in the sequential strategy, optimizing the production objective function $\left(f_{1}\right)$ does not mean systematically optimizing the global objective function (f), after PM operations insertion. This is due to the fact that a good schedule means little idles times. However, the insertion of maintenance generates a lot of shifts in the production sequence implying, thus, a deterioration of the objective function.

As a conclusion, we can say that sequential GA are better than sequential NEH and sequential TS. TS performed the worst. This is due to the fact that it starts from a random solution leading to a premature convergence.

\subsection{Integrated strategy}

In this section, we present an evaluation of the results obtained with INEH, ITS and IGA in the resolution of JPMSP by the integrated strategy. First, we present two preliminary studies on the new parameters and operators defined to solve this problem $(\S 4.3)$. In our case, no competitive results for JPMSP exist, with state-of-the-art algorithms. For this reason, we use the Taillard (1993) PFSP 20x10 benchmark problem and consider the relative increases in the common objective function yielded by IGA and ITS, relative to the best makespan value reported by the Taillard PFSP 20x10 with upper bound stated at 1659 . When comparing the solutions, the best one is the one which gave the least increase, in order to determine the best parameters to adopt. The results of the evaluation of the ITS parameters are presented in Table 3.

Table 3: Results of evaluation of the ITS parameters

\begin{tabular}{lcccc}
\hline \hline TS parameters & \multicolumn{2}{c}{ Initial solution } & \multicolumn{2}{c}{ Neighbourhood } \\
\hline \multirow{2}{*}{ Best results } & Random & Given & Production & Maintenance \\
\cline { 2 - 5 } & $21 \%$ & $79 \%$ & $43 \%$ & $57 \%$ \\
\hline \hline
\end{tabular}

ITS with given initial solution performs better. This is due to the fact that this solution is near optimal. For the neighbourhood, the strategy of shifting PM operations gives the best results. The main reason for such a superior performance is due to the fact that a PM operation has several possible insertion sites in its tolerance interval, knowing that, inside the tolerance interval, we assume that the maintenance objective function $f_{2}$ is equal to 0 .

For IGA, we executed 100 tests with the same parameters and all the crossover and mutation operators which we proposed. The results of the evaluation of the IGA operators are presented in Table 4. The following abbreviations are used:

- HC1: 1-point horizontal crossover;

\footnotetext{
${ }^{4}$ prod : production

${ }^{5} \mathrm{seq}$ : sequential strategy
} 
- HC2: 2-points horizontal crossover;

- VM1: 1-point vertical mutation;

- VM2: 2-points vertical mutation.

Table 4: Results of evaluation of the IGA operators

\begin{tabular}{|c|c|c|c|c|c|}
\hline GA operators & \multicolumn{2}{|c|}{ Maintenance crossover } & \multicolumn{3}{|c|}{ Maintenance mutation } \\
\hline Best results & $\mathrm{HC} 1$ & $\mathrm{HC} 2$ & Random & VM1 & VM2 \\
\hline & $51 \%$ & $49 \%$ & $11 \%$ & $57 \%$ & $32 \%$ \\
\hline
\end{tabular}

In the case of maintenance crossover operators, we cannot affirm that one is better than the other. The values performed by the 1-point horizontal crossover represent $51 \%$ of the best results compared to the ones with the 2ponts horizontal crossover. We think that the two operators are both technically good. On the other hand, for the mutation operators, the 1-point vertical mutation operator gave the best results. The principle of the 1-point vertical mutation which consists of mutation on the production sequence, while respecting the PM operations initial insertion sites, assumes greater significance in enhancing the performance of the obtained joint schedule.

In all experiments of the following section we will execute ITS with the parameters deduced from table 3: initial solution given, generated by constructive heuristics on which one inserts PM operation with $\mathrm{AH}+$ heuristic; and the neighbourhood is generated by shifting the PM operations. We will execute IGA with the operators deduced from table 4: 1-point horizontal crossover and 1-point vertical mutation. Table 5 presents the results obtained.

Table 5: Joint production and PM scheduling with integrated strategy

\begin{tabular}{ccccccc}
\hline & \multicolumn{3}{c}{ Production } & \multicolumn{3}{c}{ Integrated strategy } \\
\hline Benchmark & NEH & TS & GA & INEH & ITS & IGA \\
\hline $7 \times 7$ & 6590 & 6590 & 6590 & 8397 & 7397 & 6898 \\
\hline $8 \times 8$ & 8566 & 8364 & 8457 & 9628 & 10424 & 8720 \\
\hline $20 \times 5$ & 1303 & 1249 & 1318 & 1249 & 1234 & 1222 \\
\hline $20 \times 10$ & 1603 & 1602 & 1583 & 1700 & 1696 & 1686 \\
\hline $20 \times 15$ & 2002 & 2145 & 1972 & 2200 & 2200 & 2203 \\
\hline $30 \times 15$ & 2644 & 2702 & 2643 & 2859 & 2759 & 2745 \\
\hline $50 \times 10$ & 3153 & 3271 & 3218 & 3734 & 3740 & 3698 \\
\hline $75 \times 20$ & 5465 & 5497 & 5227 & 5826 & 5890 & 5851 \\
\hline $100 \times 10$ & 530 & 577 & 533 & 555 & 607 & 571 \\
\hline \hline
\end{tabular}

One notes that, overall, GA give the best results, even in the case of joint production and PM scheduling, since IGA obtained more than $60 \%$ of the best results. ITS gives $20 \%$ of the best results. However, a close look at the obtained solutions objective functions, for IGA and ITS, shows that they are very close.

In what follows, we compare NEH, TS and GA, sequential and integrated heuristics, to determine the best heuristic for the resolution of JPMSP. Tables 2 and 5 present a summary of the best performances for the sequential and integrated strategies with NEH, TS and GA. Based on values of tables 2 and 5, the values of makespan given by each heuristic are relatively evaluated against the upper bound for makespan reported by Taillard (1993) for the same benchmarks. The mean relative increases in makespan yielded by the proposed heuristics, relative to the best makespan values reported by Taillard, are noted for every problem size. The results of evaluation are presented in Table 6.

It is evident that the best values for makespan given by Taillard are better than the heuristic values yielded by the proposed algorithms. The primary reason for such a performance is that the insertion of PM operations increases the makespan values. A fair comparison of our results with the upper bound values of makespan presented by Taillard (1993) is difficult since Taillard benchmarks are dedicated to production scheduling only while ignoring PM. Moreover, the computational result of the proposed sequential and integrated algorithms are obtained with a set of non-standard test problems generated by following the procedure given by Benbouzid et al. in 2003, which represent production benchmarks proposed by Taillard(1993) combined to randomly generated maintenance benchmarks, to obtain joint production and maintenance tests problems. 
Table 6: Best variations compared to the production

\begin{tabular}{lllllll}
\hline \multirow{2}{*}{ Benchmarks } & \multicolumn{5}{c}{ Mean relative percentage increase in makespan } \\
\cline { 2 - 7 } & Production & & \multicolumn{2}{c}{ Sequential strategy } & \multicolumn{2}{c}{ Integrated strategy } \\
\hline $7 \times 7$ & 6590 & GA & 0,033 & GA & 0,030 & GA \\
\hline $8 \times 8$ & 8366 & TS & 0,062 & NEH & 0,035 & GA \\
\hline $20 \times 5$ & 1249 & TS & $-0,004$ & GA & 0,000 & GA \\
\hline $20 \times 10$ & 1603 & GA & 0,003 & GA & 0,000 & GA \\
\hline $20 \times 15$ & 1972 & GA & 0,194 & GA & 0,228 & GA \\
\hline $30 \times 15$ & 2643 & GA & 0,153 & GA & 0,102 & GA \\
\hline $50 \times 10$ & 3153 & NEH & 0,003 & GA & 0,054 & GA \\
\hline $75 \times 20$ & 5227 & TS & 0,054 & NEH & 0,056 & TS \\
\hline $100 \times 10$ & 530 & GA & 0,002 & NEH & 0,002 & NEH \\
\hline \hline
\end{tabular}

Overall, it is observed from the results that the integrated strategy perform better than the sequential one. The main reason for such a superior performance is due to the fact that the optimization in two phases of the sequential strategy (first production jobs are scheduled, which represents a strong constraint, then PM operations are inserted in the resulting schedule) decreases the quality of the obtained joint schedule. In the case of the integrated strategy, the simultaneous optimization of both production and maintenance criterion gives better results, according to a common representation of maintenance and production tasks.

\section{Conclusion and future research suggestions}

In this paper, we address the problem of joint scheduling of maintenance operations and jobs in the flowshop sequencing problem with an objective to optimise a common objective function which takes into account both production and maintenance criterion. A constructive heuristic and two efficient metaheuristics are also proposed. Moreover, we tackle the problem according to the most interesting strategies' of joint scheduling which are integrated and sequential one. Numerical experiments have resulted in the following conclusions:

- A comparative study among all the implemented methods showed that the integrated strategy is better than the sequential one because production jobs and PM operations are taken into account simultaneously.

- For the resolution by the sequential strategy, we develop heuristics for maintenance insertion. We propose IH heuristic to solve single machine problem and $\mathrm{NH}, \mathrm{AH}$ and $\mathrm{DH}$ heuristics to solve the m-machines problem. We can notice that the incidence of systematic insertion (case of heuristics $\mathrm{NH}$ with its three alternatives) is not good on joint schedule quality. Releasing this constraint, by the search of the best site for the maintenance insertion, within the boundaries of the tolerance interval (case of the heuristic $\mathrm{AH}+$ and $\mathrm{DH}+$ ), induced a clear improvement of the joint schedule quality.

- For the resolution by the integrated strategy, we propose first a new structure with two fields to represent a joint schedule. Then, for each resolution approach, we propose an adequate adaptation. Thus we adapted NEH heuristic by proposing a precedence order between both production jobs and PM operations, and finally new genetic operators for IGA and new parameters for ITS, which integrate maintenance aspect.

In all the cases, GA gave the best results then TS and NEH. This is due to the search strategy used by each method. Indeed the principle of search based on the evolutionary approach is more powerful than that of the iterative and constructive ones.

There are several directions in which this research can be extended. In this study, an assumption is that the machine does not fail. An extension can be to address both problems jointly. Some other opportunities of research may include the consideration of a stochastic behaviour. The processing times and maintenance related parameters can also be considered as random parameters following some probability distribution. The objective can be to minimize the expected value of the objective function. A multi-objective scheduling problem subjected to preventive maintenance and machine failures can also be considered for future work, as most machine scheduling problems need to satisfy more than one criterion. 


\section{Acknowledgements}

The authors wish to thank Dr. Karima Benatchba maitre de conférence at the Cole national Superior d'Informatique (ESI ex INI) of Algiers (Algeria) whose comments and insights have significantly improved the content of this paper.

\section{References}

Aggoune, R. Minimizing the makespan for the flow shop scheduling problem with availability constraints. European Journal of Operational Research, 153:534-43, 2004.

Allaoui, H., Artiba A. Integrating simulation and optimization to scheduling a hybrid flow shop with maintenance constraints. Computers and Industrial Engineering. 47:431-50; 2004.

Ashayeri, J., Teelen, A. and Selen, W. A production and maintenance planning model for the process industry. International Journal of Production Research, 34:3311-3326, 1996.

Benbouzid, F., Varnier, C., Zerhouni, N. Resolution of joint maintenance/production scheduling by sequential and integrated strategies. Proceedings of $7^{\text {th }}$ International Work Conference on Artificial and Natural Neural Networks IWANN2003 (Spain), LNCS 2687: 782-789, 2003.

Benbouzid, F., Varnier, C., Zerhouni, N. New Genetic Operator for Solving the Joint Production and Maintenance Scheduling: Application to the Flow Shop Problem. Proceedings of International Conference on Service System and Service Management ICSSSM'06 Troyes (France), 607-613, 2006.

Bennour, M., Bloch, C., Zerhouni, N. Modélisation intégrée des activités de maintenance et de production. $3^{e}$ Conférence Francophone de Modélisation et de SIMulation MOSIM'01, Troyes (France,. 805-810, 2001.

Biroloni, A. Reliability engineering, theory and practice. $4^{\text {th }}$ edition, Berlin: Springer; 2004.

Bonabeau, E, et al. Swarm Intelligence: From Natural to Artificial Systems. Oxford University Press, New York, 1999.

Brandolese, M., Fransi, M., Pozzeti, A. Production and maintenance integrated planning. International Journal of Production Research, 34(7):2059-2075, 1996.

Cassady, C.R., Kutanoglu, E. Minimizing job tardiness using integrated preventive maintenance planning and production scheduling. IIE Transactions, 35(6):503-13; 2003.

Fraternali, P., Paraboschi, S. Ordering and selecting production rules for constraint maintenance: Complexity and heuristic solution. Proceedings of IEEE Transactions on Knowledge and Data Engineering, 9(1):173178,1997

Graves, G., Lee, C.Y. Scheduling maintenance and semi-resumable jobs on single machine. Naval Research Logistics. 46:845-862, 1999.

Glover, F. Taboo search: Part I. ORSA Journal of Computing, 1:190-206, 1989.

Goldberg, D.E. Genetic algorithms in search, Optimisation and Machine learning. Addison-Wesley, Mass., 1989.

Harrath, Y., Chebel-Morello, B., Zerhouni, N. A genetic algorithm and data mining based meta-heuristic for job shop scheduling problem. Proceedings of IEEE Conference on Systems, Man and Cybernetics (Tunisia), 7:6, 2002.

Kaabi, J., Varnier, C., Zerhouni, N. Heuristics for scheduling maintenance and production on a single machine. Proceedings of IEEE Conference on Systems, Man and Cybernetics (Tunisia), 5:5, 2002.

Kaabi, J., Varnier, C., Zerhouni, N. Ordonnancement de la production et de la maintenance: cas d'un atelier de type flow shop à deux machines. APII-JESA, 37:641-660, 2003.

Lee, C.Y., Chen, Z.L. Scheduling jobs and maintenance activities on parallel machines. Naval Research Logistics, 47:145-165, 2000.

Lee, C.Y, Lin C.S. Single-machine scheduling with maintenance and repair rate-modifying activities. European Journal of Operational Research,135: 493-513, 2001.

Nawaz, N., Enscore, Jr., Ham, I. A heuristic algorithm for the m machine, n job flow shop sequencing problem, OMEGA, 11:91-95, 1983.

Nowicki, E., Smutnicki, C. A fast tabu search algorithm for the permutation flow-shop problem. European Journal of Operational Research, 91(1):160-175, 1996. 
Ponnambalam, S.G., Aravindan, P., Chandrasekaran, S. Constructive and improvement flow shop scheduling: an extensive evaluation. Production planning and control, 12 (4):335-344, 2001.

Portmann, M.C. Genetic algorithms and scheduling: a state of art and some propositions. Workshop on Production Planning and Control, I-XIV, Belgium, 1996.

Qi, X., Chen, T., Tu, F. Scheduling the maintenance on single machine. Journal of the Operational Research Society, 50(10):1071-1078, 1999.

Raza, S.A., Al-Turki, U.M. and Selim, S.Z. Early-tardy minimization for joint scheduling of jobs and maintenance operations on a single machine. International Journal of Operations Research, 4(1):32-41, 2007.

Rinnooy Kan, AHG. Machine scheduling problems: classification, complexity and computations. The Hague: Martinus Nijhoff; 1976.

Ruiz, R., García-Díaz, J.C, Maroto, C. Considering scheduling and preventive maintenance in the flowshop sequencing problem. Computers and Operations Research, 34:3314 - 3330; 2007.

Sanmarti, E., Espuna, A., Puigjaner, L. Batch production and preventive maintenance scheduling under equipment failure uncertainty. Computer Chemical Engineering, 21(10):1157-1168, 1997.

Sloan, T.W., Shanthikumar, J.G. Combined production and maintenance scheduling for a multiple product, single machine production system. Production and Operation Management, 9(4):379-399, 2000.

Sortrakul, N., Nachtmann, H., and Cassady, C.R. Genetic algorithms for integrated preventive maintenance planning and production scheduling for a single machine. Computers in Industry, 56: 161-168, 2005.

Taillard, E. Some efficient heuristic methods for the flow shop sequencing problem. European Journal of Operational Research, 47:65-74, 1990.

Taillard, E. Benchmarks for basic scheduling problems. European Journal of Operational Research, 64:278285, 1993. Available from: http://ina.eivd.ch/Collaborateurs/etd/default.htm. 